\title{
Correction: Targeting the a4-a5 dimerization interface of K-RAS inhibits tumor formation in vivo
}

\author{
Imran Khan ${ }^{1,2,3,4} \cdot$ Russell Spencer-Smith ${ }^{1,2} \cdot$ John P. O'Bryan ${ }^{1,2,3,4}$
}

Published online: 1 February 2019

c) Springer Nature Limited 2019

\section{Correction to: Oncogene;}

https://doi.org/10.1038/s41388-018-0636-y;

Published online 20 December 2018

The original version of this article contained errors in the author affiliations which were originally listed as: ${ }^{2}$ Department of Cell, Jesse Brown VA Medical Center, Chicago, IL, USA. ${ }^{3}$ Molecular Pharmacology and Experimental Therapeutics, Hollings Cancer Center, Medical University of South Carolina, Charleston, SC 29425, USA. and have now been corrected to: ${ }^{2}$ Jesse Brown VA Medical Center, Chicago, IL 60612 USA. ${ }^{3}$ Department of Cell and Molecular Pharmacology and Experimental Therapeutics, Hollings Cancer Center, Medical University of South
Carolina, Charleston, SC 29425, USA. This correction has been made in both the PDF and HTML versions of the article.

Additionally, a further error has been corrected on page five in the sentence: Subsequent improvements to the chemistry of these lead compounds have resulted in the most recent interaction, with ARS-1620, which demonstrates selective inhibition of K-RAS(G12C) mutant tumor models in vivo [23]. The word 'with' has been removed from this sentence to ensure the correct meaning is communicated, such that the sentence now is: Subsequent improvements to the chemistry of these lead compounds have resulted in the most recent interaction, ARS-1620, which demonstrates selective inhibition of K-RAS(G12C) mutant tumor models in vivo [23].

These authors contributed equally: Imran Khan, Russell SpencerSmith

The original article can be found online at https://doi.org/10.1038/ s41388-018-0636-y.

John P. O'Bryan

obryanjo@musc.edu

1 Department of Pharmacology, University of Illinois Cancer Center, University of Illinois at Chicago, Chicago, IL 60612, USA

2 Jesse Brown VA Medical Center, Chicago, IL 60612, USA

3 Department of Cell and Molecular Pharmacology and Experimental Therapeutics, Hollings Cancer Center, Medical University of South Carolina, Charleston, SC 29425, USA

4 Ralph H. Johnson VA Medical Center, Charleston, SC 29401, USA 